\title{
The development and evaluation of a self-marking unit to estimate malaria vector survival and dispersal distance
}

\author{
Adam Saddler ${ }^{1,2,3^{*}}$ (D, Katharina S. Kreppel ${ }^{1,4}$, Nakul Chitnis ${ }^{2,3}$, Thomas A. Smith ${ }^{2,3}$, Adrian Denz ${ }^{2,3}$, \\ Jason D. Moore ${ }^{1,2,3}$, Mgeni M. Tambwe $e^{1,2,3}$ and Sarah J. Moore ${ }^{1,2,3}$
}

\begin{abstract}
Background: A clear understanding of mosquito biology is fundamental to the control efforts of mosquito-borne diseases such as malaria. Mosquito mark-release-recapture (MMRR) experiments are a popular method of measuring the survival and dispersal of disease vectors; however, examples with African malaria vectors are limited. Ethical and technical difficulties involved in carrying out MMRR studies may have held back research in this area and, therefore, a device that marks mosquitoes as they emerge from breeding sites was developed and evaluated to overcome the problems of MMRR.
\end{abstract}

Methods: A modified self-marking unit that marks mosquitoes with fluorescent pigment as they emerge from their breeding site was developed based on a previous design for Culex mosquitoes. The self-marking unit was first evaluated under semi-field conditions with laboratory-reared Anopheles arabiensis to determine the marking success and impact on mosquito survival. Subsequently, a field evaluation of MMRR was conducted in Yombo village, Tanzania, to examine the feasibility of the system.

Results: During the semi-field evaluation the self-marking units successfully marked $86 \%$ of emerging mosquitoes and there was no effect of fluorescent marker on mosquito survival. The unit successfully marked wild male and female Anopheles gambiae sensu lato (s.l.) in sufficiently large numbers to justify its use in MMRR studies. The estimated daily survival probability of An. gambiae s.l. was $0.87(95 \% \mathrm{Cl} 0.69-1.10)$ and mean dispersal distance was $579 \mathrm{~m}$ (95\% Cl 521-636 m).

Conclusions: This study demonstrates the successful use of a self-marking device in an MMRR study with African malaria vectors. This method may be useful in investigating population structure and dispersal of mosquitoes for deployment and evaluation of future vector control tools, such as gene drive, and to better parameterize mathematical models.

Keywords: Mark-release-recapture, Anopheles, Mosquito, Vector, MMRR, Release-recapture, Dispersal, Survival

\section{Background}

A clear understanding of mosquito biology is fundamental to the effective control of mosquito-borne diseases including

*Correspondence: adam.saddler@swisstph.ch

1 Ifakara Health Institute, Environmental Health and Ecological Sciences, P.O. Box 74, Bagamoyo, Tanzania

Full list of author information is available at the end of the article malaria, dengue, Zika and lymphatic filariasis. While novel vector control tools such as spatial repellents [1, 2], attractive targeted sugar baits (ATSBs) [3, 4] and gene-drive systems [5], continue to be developed, our understanding of several key aspects of mosquito biology remains limited. Therefore, predicting how these novel tools will function in real world settings is difficult. Planning and evaluation of these new

(c) The Author(s) 2019. This article is licensed under a Creative Commons Attribution 4.0 International License, which permits use, sharing, adaptation, distribution and reproduction in any medium or format, as long as you give appropriate credit to the original author(s) and the source, provide a link to the Creative Commons licence, and indicate if changes were made. The images or other third party material in this article are included in the article's Creative Commons licence, unless indicated otherwise in a credit line to the material. If material is not included in the article's Creative Commons licence and your intended use is not permitted by statutory regulation or exceeds the permitted use, you will need to obtain permission directly from the copyright holder. To view a copy of this licence, visit http://creativeco mmons.org/licenses/by/4.0/. The Creative Commons Public Domain Dedication waiver (http://creativecommons.org/publicdomain/ zero/1.0/) applies to the data made available in this article, unless otherwise stated in a credit line to the data. 
tools, in particular gene drives, require a detailed understanding of mosquito dispersal and survival of both male and female mosquitoes [6].

Mosquito mark-release-recapture (MMRR) studies have been one of the most widely used ways to obtain field estimates of daily mosquito survival, population size, duration of the gonotrophic cycle and dispersal distances $[7,8]$, since tracking individual mosquitoes over distances larger than a few metres remains infeasible. A review in 2014 of MMRR studies with female mosquitoes identified 774 separate MMRR experiments covering 58 mosquito species that are of importance for human disease transmission [8]. However, there is a paucity of studies on African malaria vectors with only 11 studies on Anopheles gambiae sensu lato (s.l.) identified. Daily survival is the most studied parameter in MMRR studies on malaria vectors [9-12], although population estimates $[6,11,13,14]$ and behavioural studies [15-17] are also conducted. Detailed analysis of dispersal distances were carried out by Gillies with An. gambiae in Tanzania [9] and Costantini et al. [18] with An. gambiae s.l. in Sudan. Considering the burden of malaria across Africa and the variety of ecological settings across the continent, there are surprisingly few field-estimates of the essential entomological parameters for optimizing the implementation of focused vector control [19] and the design of cluster randomized field trials [20].

The numerous ethical and technical difficulties involved in carrying out MMRR studies have been described in detail $[7,21]$ and guidance on the safety of MMRR studies is available [22]. The methods used in each step of an MMRR study, from sourcing, marking, releasing, recapturing and handling mosquitoes, has the potential to disrupt the normal behaviour and survival of the wild mosquitoes. Visual markers such as fluorescent powders and paints have been the main method to mark anopheline mosquitoes $[8,21]$, but reports on their impact on mosquito survival are inconclusive [21, 23-25]. This variation in results may be caused by species or environmental differences but is most likely due to the different application methods, the degree of mosquito handling, the amount and brand of pigment applied [25]. It is therefore important to measure the impact of the marking method on survival when using a new marker and species combination.

At the forefront of ethical concerns is the risk of releasing highly competent, potentially disease transmitting, mosquitoes and so careful consideration is needed for a study to be carried out safely [22]. It could be argued that as MMRR studies capture far more mosquitoes than they release they mitigate any risk of increasing the local vector population in the short-term [22]. However, MMRR studies that release laboratory strains of mosquitoes have potential longer-term risks. Laboratory strains are often selected for increased longevity, strong human feeding preference or high susceptibility to parasite infection. All of these characteristics could dramatically increase vector competence if expressed in wild vector populations through inter-breeding with released individuals. In addition to the ethical concerns, using laboratory reared mosquitoes with different survival rates and dispersal behaviours to their wild counterparts will misrepresent the very parameters that are being estimated [26].

Sourcing mosquitoes from wild populations is preferable, and is in fact the most common method used in MMRR studies with Anopheles [8], but it has its own limitations. Collection of large numbers of mosquitoes is required for MMRR studies, but the capturing and handling of adult mosquitoes before marking may cause stress and consequently impact on their survival and dispersal. Furthermore, information on mosquito age or infection status is unknown at the time of marking and may influence results.

In order to overcome these disadvantages a self-marking unit first developed by Niebylski and Meek to mark Culex quinquefasciatus [27] as they emerge from the breeding sites was adapted and optimized for use with Anopheles arabiensis, Anopheles funestus, and Anopheles gambiae sensu stricto (s.s.).

\section{Methods}

Study site

The marking unit was first evaluated in the Ifakara Tunnel, a large screened cage at the Bagamoyo branch of the Ifakara Health Institute (IHI), Tanzania [28]. The tunnel provides ambient environmental conditions where experiments can be conducted safely with disease-free laboratory-reared mosquitoes. Field evaluations were conducted at Yombo village, Tanzania $\left(6^{\circ} 35^{\prime} 01.0^{\prime \prime} \mathrm{S}, 38^{\circ}\right.$ $50^{\prime} 48.4^{\prime \prime}$ E, Fig. 1) located approximately $17 \mathrm{~km}$ south of Bagamoyo town and $5 \mathrm{~km}$ east of the Ruvu river in the Pwani region of Tanzania. Bagamoyo district experiences an annual rainfall of $800-1000 \mathrm{~mm}$ and an average temperature of $28{ }^{\circ} \mathrm{C}$. Two rainy seasons replenish permanent breeding sites such as streams and ponds and create temporary breeding sites such as puddles. Malaria is endemic in Bagamoyo and the main vectors are Anopheles arabiensis, An. funestus, and An. gambiae s.s. [29].

\section{Experimental design}

Experiments to optimize the self-marking unit were first conducted under semi-field conditions to determine (i) the efficiency of the self-marking unit measured as the percentage of marked mosquitoes; (ii) if marking had an impact on survival; and (iii) if pigment transfer to unmarked mosquitoes occurred during mosquito collection. Experiments were conducted using 


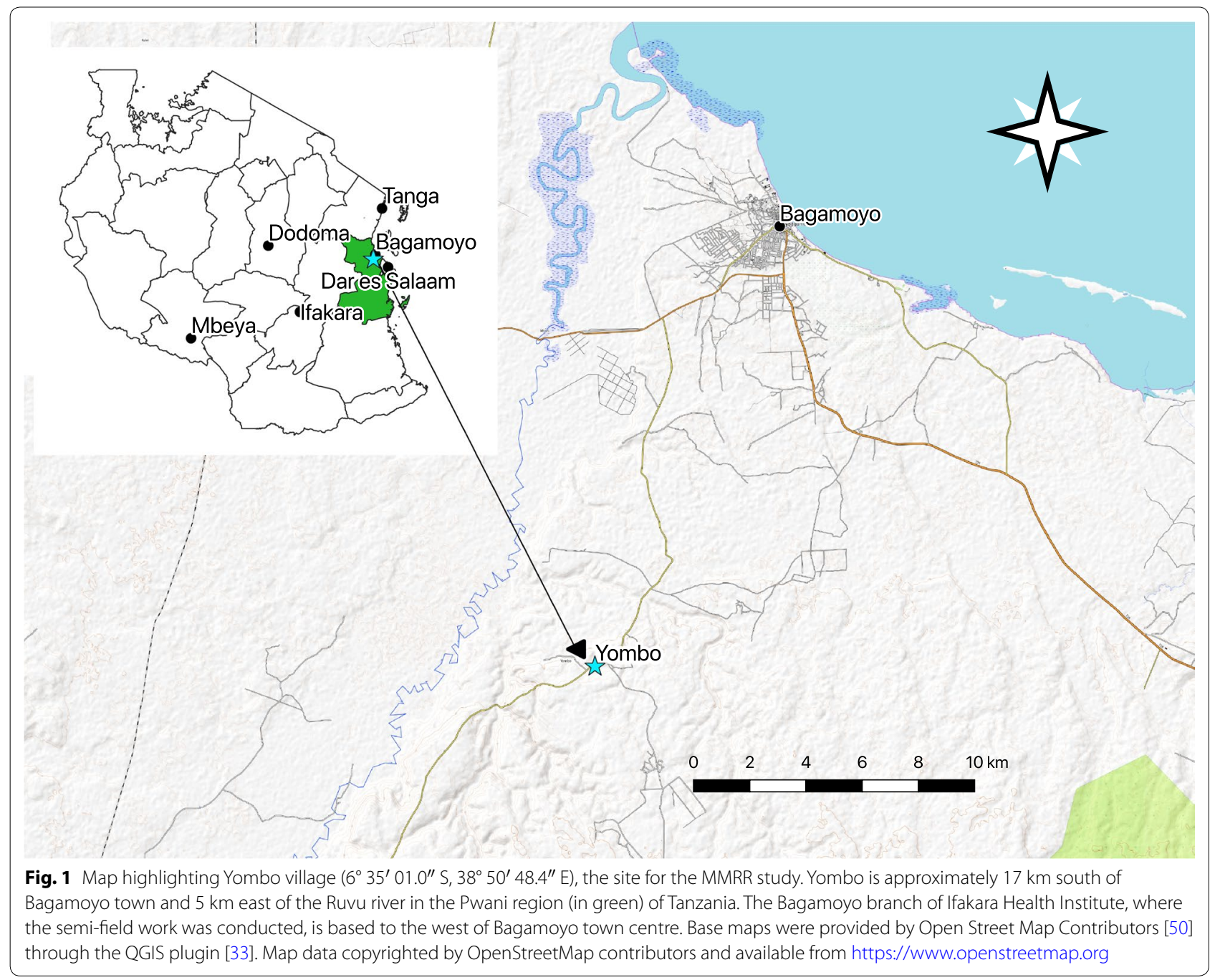

An. arabiensis (Kingani strain) reared under standard laboratory conditions previously described [30].

This was followed by preliminary field experiments in breeding sites located close to the IHI, Bagamoyo Branch to measure the number of mosquitoes marked by the self-marking units. Marking units covering natural breeding sites were compared to units which were placed next to the breeding site and had pupae placed in bowls underneath them from the surrounding breeding site. Finally, to test the feasibility of the self-marking unit and possible applications in studies with wild malaria vectors, a small MMRR field trial was conducted in Yombo Village.

\section{Self-marking unit design}

The core component of the self-marking unit is the marking grid containing cloth impregnated with fluorescent pigment (Fig. 2). As adult mosquitoes emerge from pupae and they must pass through the layers of impregnated cloth to take their first flight and as a result are marked with the pigment. To allow daily colour change the unit was designed so that the marking grid can easily be removed and replaced with a new grid containing a different colour of fluorescent. Five colours from the A series range of fluorescent pigments were selected for the study: Laser Red 3, Flame Orange 4, Solar Yellow 7, Stellar Green 8 and Comet Blue 80 (SWADA, Cheshire, UK). For the remainder of the manuscript the colours will be referred to as pink, orange, yellow, green and blue, respectively. White loose weave cotton cloth was purchased from a local fabric store and cut into $50 \times 50 \mathrm{~cm}^{2}$. The cloth was placed in a large plastic bag with half a cup of fluorescent pigment (approx. $125 \mathrm{~g}$ ) and shaken until an even coating of colour was achieved. 


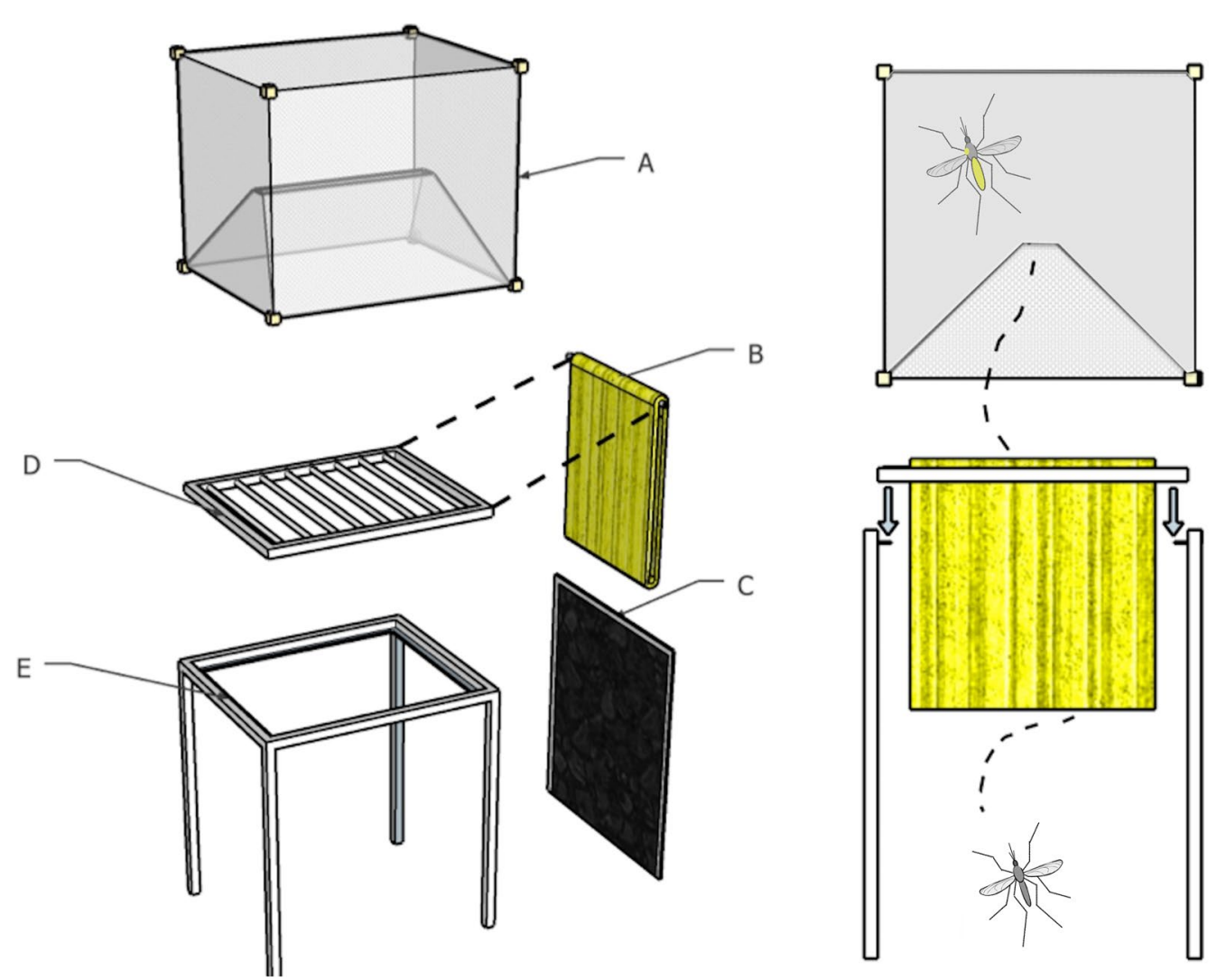

Fig. 2 The self-marking unit adapted from Niebylski and Meek [27]. Left panel: 3D model of the marking unit indicating the key components. A - an exit trap used previously for hut trials as a window trap [31]. A slit in the netting allows mosquitoes to pass through in one direction thus collecting the mosquitoes after they have passed through the marking unit. The exit traps were only used when marked mosquitoes need to be retained as in the semi-field experiments, B — cloth impregnated with fluorescent pigment, C—black cloth side panels attached to the frame with Velcro, D-detachable marking grid from which the impregnated cloth hangs. It can be removed without tools and replaced with another grid containing a new colour, E-frame to hold the marking grid made from $2 \times 2 \mathrm{~cm}^{2}$ metal tubing. Right panel: side view of the unit to show the frames' internal lip on which the marking grid sits. The path of an emerging mosquito is shown passing through the marking grid and picking up fluorescent pigments

\section{Marking grid dimensions}

The marking grid was made from $2 \times 2 \mathrm{~cm}$ wide, square metal tubing and measured $54.5 \times 45 \mathrm{~cm}$ with interspersed metal rods at $5 \mathrm{~cm}$ intervals spanning the $45 \mathrm{~cm}$ width. Impregnated cloth was attached to the grid by looping a $50 \times 50 \mathrm{~cm}$ piece of cloth around one rod and by stapling the ends of the cloth together at the bottom - this was done for all eight rods of the marking grid. The frame to hold the marking grid was also made from $2 \times 2 \mathrm{~cm}^{2}$ metal tubing and measured $59 \times 49.5 \mathrm{~cm}$ with $62 \mathrm{~cm}$ long legs. An inner lip of metal sheeting measuring $2 \mathrm{~cm}$ (Fig. 2) allowed the marking exit grid to sit snugly within the frame without the use of tools for attachment.

Black cloth was attached with Velcro to all sides of the frame to enclose the unit and to ensure that the only exit was up through the impregnated cloth. Preliminary experiments indicated that dark cloth that fitted tightly around the frame increased exiting rates of laboratory reared $A n$. arabiensis from the units compared to netting or a loose-fitting funnel shape. The dimensions of the marking units allowed the attachment of exit traps previously designed for trapping mosquitoes exiting windows in hut studies [31] (Figs. 2, 3). The exit traps were used in both semi-field and field experiments to capture mosquitoes exiting the marking units and thus enabling mosquito collection and the examination of pigment transfer to the mosquitoes.

\section{Marking success and survival of laboratory reared Anopheles arabiensis}

Six self-marking units with exit traps attached were placed in a large experimental chamber $(5 \mathrm{~m} \times 3 \mathrm{~m} \times 2.1 \mathrm{~m})$ under semi-field conditions. Each unit contained a different colour of fluorescent marker (orange, blue, yellow, green, pink) or a pigment-free cloth as a control (Fig. 3). At 17:00 h East African Time (EAT), 60 laboratory reared An. arabiensis pupae, in a bowl with water, were placed under the self-marking unit and left overnight to emerge. 


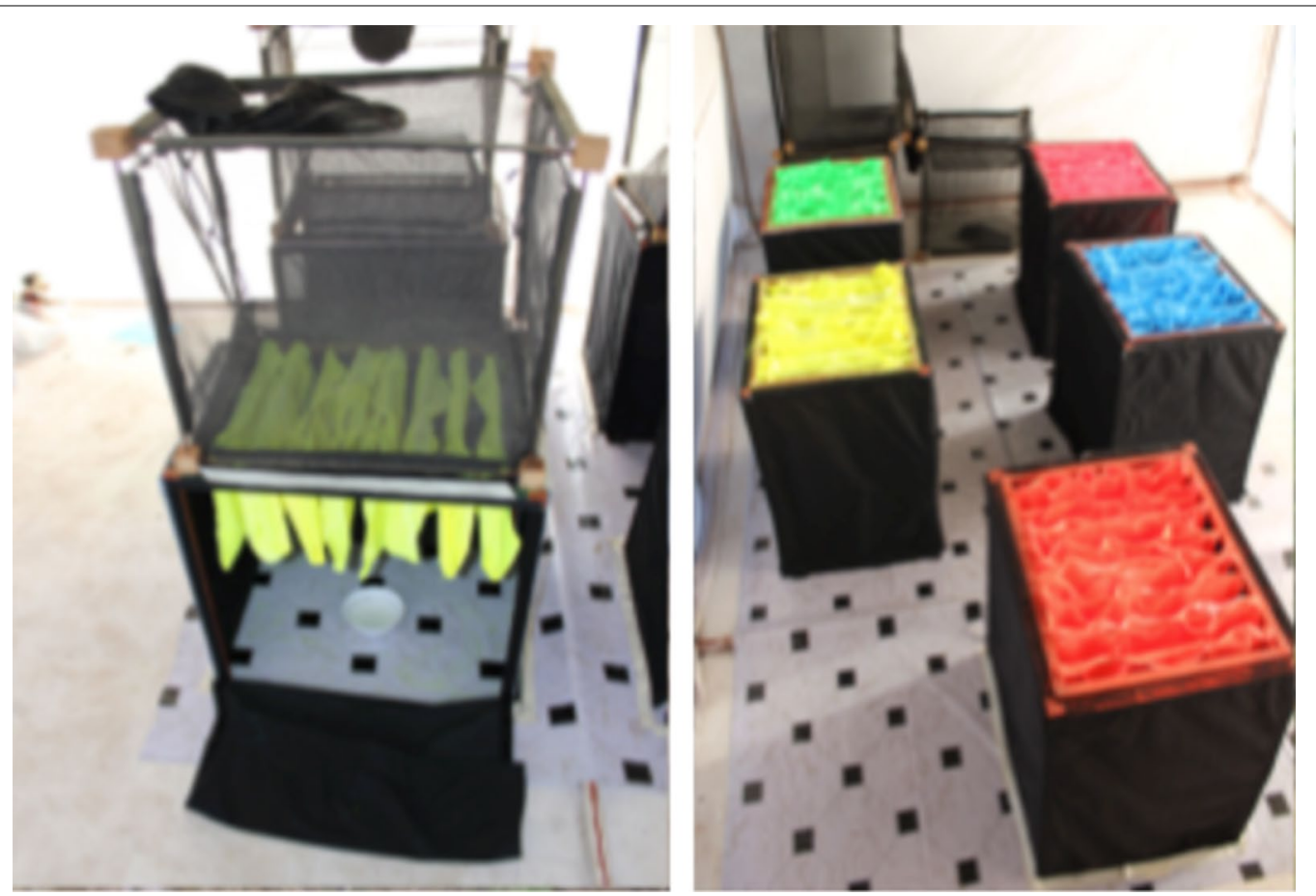

Fig. 3 Evaluation of the self-marking units under semi-field conditions. Left panel: an open side panel showing a bowl of An. arabiensis pupae underneath. The side panel is closed for the experiment and emerging adult mosquitoes fly and bump their way through the layers of cloth to exit the marking grid and into the exit trap. Adults are collected from the exit trap by aspiration through the cloth sleeve on top of the trap. Right panel: the units set-up as intended for field use with side panels closed and exit traps removed. Five units each containing a different colour marker used in the study

At 10:00 h EAT the following day, mosquitoes that had emerged and had been captured in the exit traps were transferred to holding cups and provided with cotton wool soaked in $10 \%$ sucrose solution. Any pupae that had not emerged and remained in the bowl under the marking unit were left for a further $24 \mathrm{~h}$ for a second collection from the exit traps. The marking process was repeated four times over 8 days. Holding cups were labeled with a unique individual identification number that identified the exit trap, colour pigment and date of emergence. Each holding cup contained a maximum of twenty mosquitoes and was immediately transferred to a screened laboratory and held at ambient temperature and humidity with access to sugar. Survival of the mosquitoes was recorded daily until all mosquitoes were dead. Each day all dead mosquitoes were removed and checked for florescent powder using a UV-torch and microscope.

\section{Pigment transfer during recapture of laboratory Anopheles arabiensis using CDC light traps or aspiration}

The self-marking device relies on mosquitoes picking up fluorescent pigment when they contact impregnated cloth. It is therefore not unreasonable to assume that mosquitoes could also pick up the pigment when they contact mosquitoes that already have been marked especially when forced into the confined space of traps and collection tools. Pigment transfer during collection was assessed for three common methods of mosquito sampling: the Centers for Disease Control and Prevention light trap (CDC-LT), the battery powered Prokopack aspirator [32] and standard mouth aspirator.

Five CDC-LTs were hung individually in five large cages $(120 \times 120 \times 120 \mathrm{~cm})$. At 18:00 h EAT, 20 mosquitoes were introduced to each cage: 10 mosquitoes that were marked using the self-marking unit and 10 unmarked mosquitoes. The traps were left to run overnight and the number of mosquitoes with colour pigment was assessed the following day. If there were more than 10 marked mosquitoes then it was deemed pigment transfer had taken place. Due to the size of the cages, the CDC-LTs did not use an odour-lure as the phototactic response was sufficient to attract the majority of released mosquitoes to the trap. However, any mosquitoes not in the trap and still in the cage were adjusted for when recording results. Five replicates of each of the five colors were conducted over five nights.

A similar method was used to examine pigment transfer while using a Prokopack or manual aspiration. Five marked and five unmarked mosquitoes were released into each of the large cages. Collections were 
conducted $10 \mathrm{~min}$ after the mosquitoes were released. Two manual aspiration methods were examined; (i) aspiration of mosquitoes individually but transferred to the same cup (ii) group aspiration (3-5 mosquitoes at a time) before transferring to the same cup. Again, five replicates were conducted for each colour pigment. All replicates were completed in a single day after which they were transferred to the laboratory for counting (1-2 $\mathrm{h}$ after collection).

\section{Field testing \\ Preliminary trials in natural breeding sites and the development of a pupae collection method}

The original self-marking device for Culex quinquefasciatus mosquitoes was designed to be used over natural breeding sites [27]. As the breeding sites of mosquito species can vary significantly, several different prototypes were developed to cover the breeding sites of Anopheles arabiensis, An. funestus, and An. gambiae s.s. The basic marking unit covers a breeding site of $55 \mathrm{~cm} \times 45 \mathrm{~cm}$ and therefore it can fit over small temporary breeding sites favoured by An. gambiae, such as water-filled hoof prints or puddles. The Velcro side panels were removed and prototypes with tarpaulin skirt extensions were made to cover ditches and larger breeding sites. A floating unit was designed in order to mark An. arabiensis, which is often found in rice paddies, and An. funestus, often located in more permanent water bodies like swamps and ponds. A method to increase the mosquito numbers passing through the marking unit was also tested by collecting pupae and stage four larvae from the surrounding breeding site and placing them in a bowl under the unit.

Five of the basic marking units were deployed for 10 days over a breeding site close to IHI Bagamoyo Branch and trapped emerging mosquitoes in attached exit traps (Fig. 4). For five of these days, the units were deployed on natural breeding sites and for the other 5 days, the devices were deployed in the same area but contained small bowls underneath, where pupae and stage four larvae were placed after collection by the field team.

\section{MMRR field trial}

\section{Estimation of released mosquitoes}

A productive breeding site for Anopheles mosquitoes was identified in Yombo and one marking unit was placed adjacent to the breeding site for 5 days. On each day, a marking grid with a new colour was introduced. Trained technicians collected pupae from an area within $20 \mathrm{~m}$ of the marking unit for approximately $1 \mathrm{~h}$ each day. As species identification of pupae can be difficult, breeding sites were sampled where predominantly Anopheles larvae were found. The collected pupae were counted and placed in a bowl under the marking system at 18:00 $\mathrm{h}$ EAT. The following day, the pupae remaining in the bowl were counted and subtracted from the previous day's total to calculate the number of mosquitoes that had emerged through the marking grid.

\section{Recapture methods}

Adult mosquito collections were conducted for 12 days, following the first marking day, among thirty houses upon written informed consent of the household head. GPS coordinates of each household were taken to calculate the distance and direction of the household in relation to the marking unit using QGIS software version 3.6.0 [33]. No other household information was recorded. To maximize recapture probability, recapture was focused in an area within $1 \mathrm{~km}$ of the breeding site (Fig. 5). Outdoor resting mosquitoes were sampled from all 30 households with resting buckets (RBu) [34] and indoor host seeking mosquitoes were sampled among 20 of the 30 households using CDC-LTs.

Ten RBu were deployed at each sampling site and were placed facing the household roughly five metres away in all directions. Mosquitoes were collected at 06:00 each morning using a Prokopack aspirator [32]. The sum of mosquitoes caught in the ten buckets was considered the $\mathrm{RBu}$ catch for 1 day for that household. CDC-LTs were deployed from between approximately 18:00 $\mathrm{h}$ and 06:00 h every night by hanging them approximately $1.5 \mathrm{~m}$ above ground and close to the foot of a bed in which an individual slept under an insecticide-treated bed net. CDC-LT catch bags were collected in the morning shortly after the resting buckets were sampled. Mosquito identification and inspection of each mosquito for fluorescent pigment was performed daily using UV-torch and microscope.

\section{Data management and statistical analysis}

All data were collected first by hard copy and then transferred into Excel using double entry. Analyses were carried out with R statistical software v3.5.2 [35].

\section{Survival analysis of laboratory Anopheles arabiensis}

Mosquito survival was measured in days and analysed with a mixed-effects Cox model using the "coxme" package in R [36]. Colour was included in the model as a fixed factor with 6 levels (5 colours and control). Night of emergence and mosquito sex were also included as fixed factors. Round and cup ID number were included as random factors. From the mosquitoes that passed through the coloured marking grids, only mosquitoes identified 


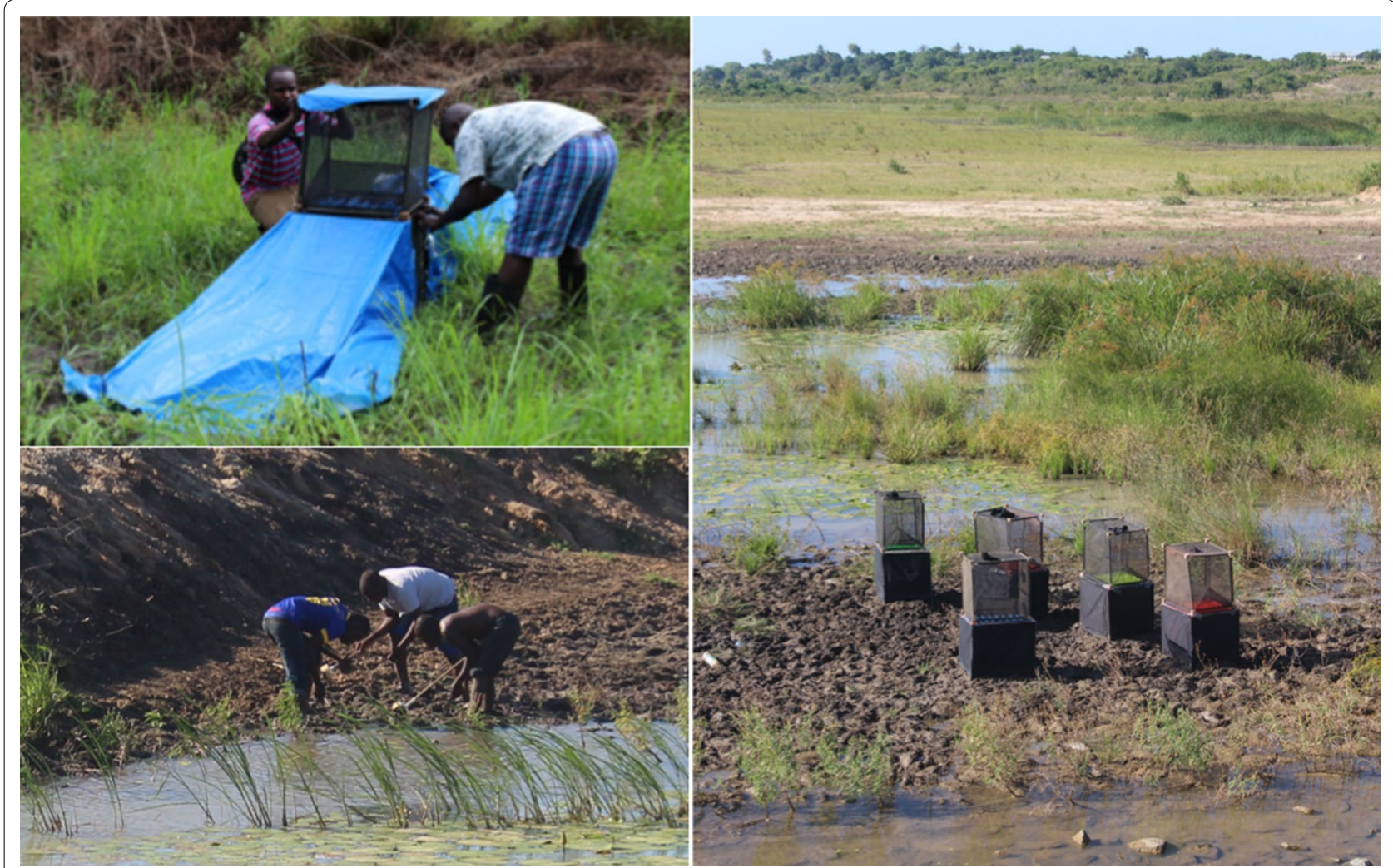

Fig. 4 Self-marking units in the field with exit traps to measure exiting rates of mosquitoes from natural breeding sites and sites were the numbers of pupae were manipulated. Top left: self-marking UNIT with tarpaulin skirt extension to cover a ditch where Anopheles larvae were found. Right: five basic self-marking units containing the five pigments used in the study (pink, orange, yellow, green and blue). Bottom left: pupae collection with dippers. Larval dippers were first used to identify productive breeding sites and then to collect pupae to be placed under the self-marking devices

as with a colour pigment were included in the survival analysis.

\section{Marking success of laboratory Anopheles arabiensis}

Marking success was determined by the percentage of mosquitoes marked after passing through the marking unit and was analyzed using binomial Generalized Linear Mixed Effects Models (GLMM). The colour of the pigment, mosquito sex and emergence day were treated as fixed factors and round of experiment was included as a random factor. An individual random effect was included in the model to account for overdispersion after it was identified in the initial models. The analysis was carried out using the "lme4" package [37]. Post hoc pairwise comparisons using Tukey contrasts were preformed between each colour pigment using the "multcomp" package [38].

\section{MMRR field trial}

Summary statistics were used to describe the number of mosquitoes marked by the units and the total number of marked and unmarked mosquitoes captured during the trapping. Data from the mark-recaptured mosquitoes was used to calculate daily survival rates and mean distance travelled (MDT) by An. gambiae s.l.

The MDT was calculated using a correction factor that takes account of uneven sampling effort over distance [39, 40]. Briefly, the sampling area was divided into four concentric annuli separated by $200 \mathrm{~m}$. For each annulus the number of traps and area were used to calculate a correction factor $(\mathrm{CF})$,

$$
\text { AnnulusCF }=\frac{\text { Area of annulus }}{\text { Total trapping area }} \times \text { total number of traps. }
$$

The correction factor was then applied to the observed recapture numbers in order to calculate the estimated recapture (ER) per annulus,

$$
\begin{aligned}
E R= & \frac{\text { Number of observed recaptures in annulus }}{\text { Number of traps in annulus }} \\
& \times \text { AnnulusCF. }
\end{aligned}
$$

Finally the MDT was calculated as 

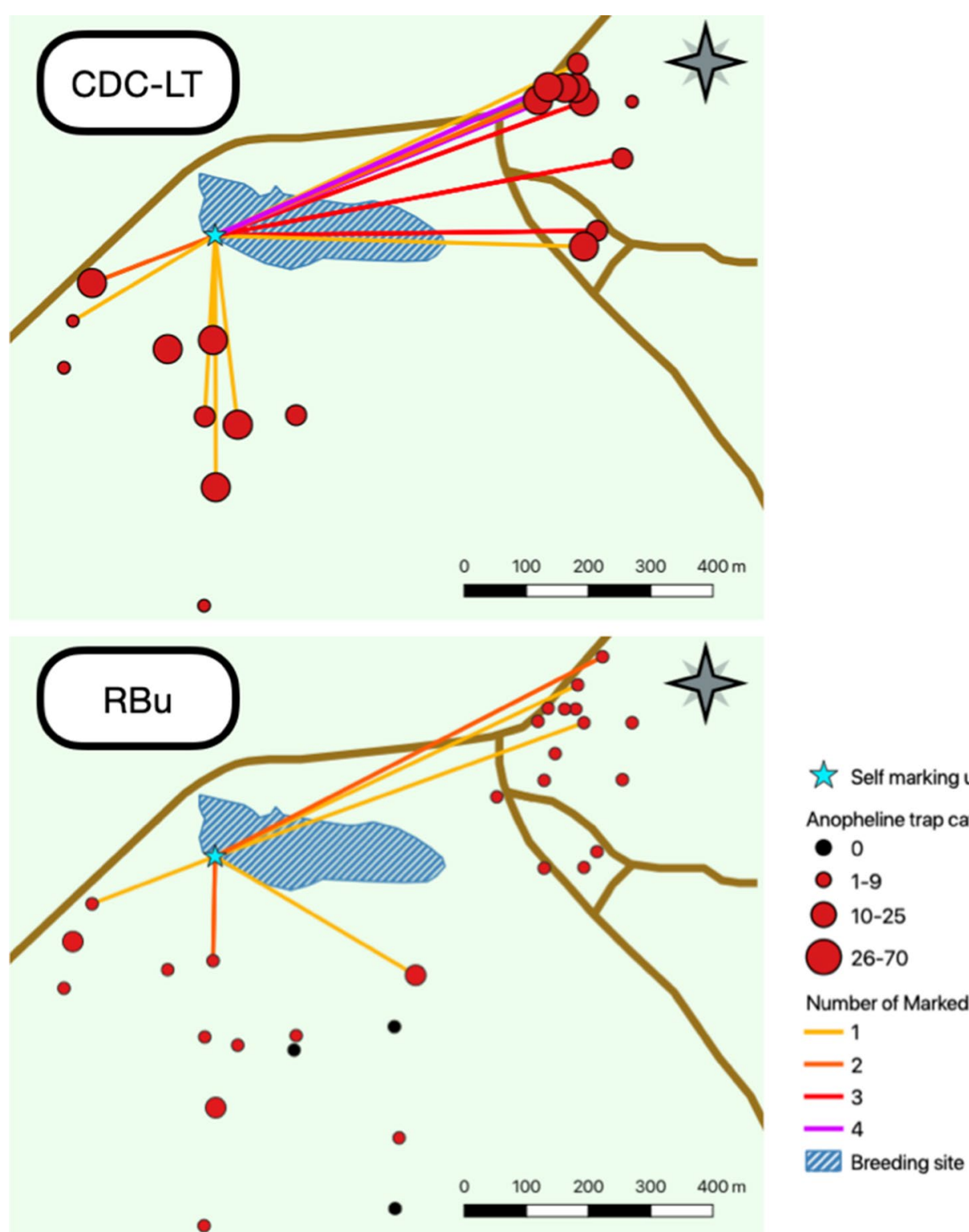

Fig. 5 Distribution of marked and unmarked female Anopheline mosquitoes caught by CDC light traps (top) or resting bucket traps (bottom). Size and colour of circles indicate the total number of female anopheline mosquitoes (unmarked and marked) caught in each trap for the duration of the trapping ( 12 days). Marked and recaptured mosquitoes are indicated by lines dispersing from the self-marking unit-also indicating the total number of marked mosquitoes caught at the final trap location

$$
M D T=\frac{\sum(\text { AnnulusER } \times \text { annulus distance }) \text { for all annuli }}{\text { Total number of } E R} .
$$

The MDT was first calculated using recaptured mosquitoes from all 12 days of recapture; a "first flight" MDT was then calculated using only mosquitoes recaptured in the 3 days following marking. The calculation of MDT makes simplifications because it treats each location as equally attractive to mosquitoes, and assumes dispersal is equal in all directions.
The daily survival probability was estimated using an exponential model [9], where the $\log _{10}(x+1)$ number of recaptures is regressed against day after marking and the antilog $_{10}$ of the slope of the regression is the daily survival probability. Average life expectancy (ALE) was derived from the survival estimate [41].

\section{Results}

\section{Survival of laboratory Anopheles arabiensis}

The daily survival of marked mosquitoes in the laboratory was not significantly different from unmarked 
mosquitoes independent of the pigment colour; Blue $(\mathrm{HR}=1.12,95 \%$ CI $0.81-1.43, \mathrm{p}=0.48)$, Green $(\mathrm{HR}=1.34, \quad 95 \% \quad \mathrm{CI} \quad 0.98-1.68, \quad \mathrm{p}=0.10)$, Orange $(\mathrm{HR}=1.02,95 \%$ CI $0.71-1.35, \mathrm{p}=0.91)$, Pink 0.97, 95\% CI $0.64-1.30, \mathrm{p}=0.87)$ and Yellow $(\mathrm{HR}=1.19,95 \% \mathrm{CI}$ $0.86-1.52, \mathrm{p}=0.30$ ) (Fig. 6). With a hazard ratio of 1.67 (95\% CI 1.51-1.83, $\mathrm{p}<0.001$ ), male mosquitoes were found to be $67 \%$ more likely to die than females each day. Mosquitoes that emerged on the second night of marking also had increased daily mortality risk $(\mathrm{HR}=1.52,95 \% \mathrm{CI}$ $1.32-1.72, \mathrm{p}<0.001)$.

\section{Marking success of laboratory Anopheles arabiensis}

On average, $85.9 \%$ (CI 82.5-87.9\%) of An. arabiensis emerging in the laboratory experiments were marked. Yellow pigment was successfully transferred to $98.3 \%$ (CI 93.3-99.7\%) of mosquitoes passing through the marking grid compared to $88.6 \%$ (CI 80.5-93.7\%) with green pigment, $84.8 \%$ (CI 78.5-89.6\%) with orange pigment, $82.8 \%$ (CI 75.6-88.2\%) with blue pigment and 75\% (CI 66.4-82.0\%) with pink pigment. Tukey contrast indicated the only significant differences in the marking success were between yellow and three of the other four colours; pink $(\mathrm{p}<0.001)$, orange $(\mathrm{p}=0.011)$, and blue $(\mathrm{p}=0.004)$. Mosquitoes emerging on the second day had a higher overall marking success with $87.9 \%$ (CI $84.0-91.0 \%$ ) marked compared with $82.4 \%$ (CI $77.5-86.4 \%)$ on the first night $(\mathrm{p}=0.011)$. The mosquitoes emerging on the second day may have spent longer under the marking unit and spent the daylight hours resting on the impregnated cloth, which could explain the higher marking rate. There was no difference in the

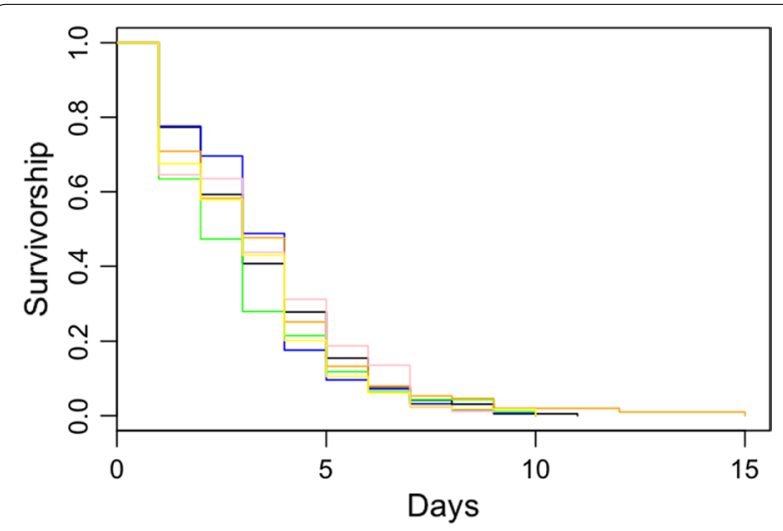

Fig. 6 Kaplan-Meier survival curves of laboratory reared An. arabiensis mosquitoes marked with the self-marking unit. Five colours (blue, pink, yellow, orange and green) were examined for their impact on mosquito survival. The colours of the lines represent mosquitoes marked with that colour. The black survival curve is from unmarked controls. There was no significant impact of individual colours or marking as a whole marking rate of male $(84.8 \%$, CI $80.1-88.6 \%)$ and female mosquitoes (85.8\%, CI 81.8-89.1\%) ( $\mathrm{p}=0.52)$.

The number of mosquitoes marked during the MMRR field trial was calculated using a correction factor based on the average marking success in this semifield experiment. While an individual correction factor could be applied for each colour, it was decided that the average of all colours (85.9\%) was to be used as only the yellow pigment showed differences in marking success in select comparisons.

\section{Pigment transfer experiments}

During the controlled pigment transfer experiment, transfer of pigment was not observed when collecting mosquitoes with CDC light traps (0/25 trials), Prokopacks $(0 / 25$ trials $)$ or when aspirating mosquitoes individually ( $0 / 25$ trials). However, pigment transfer was observed in $2 / 25$ trials $(8 \%)$ when aspirating mosquitoes in groups. In both of the two trials where pigment transfer was observed only one additional mosquito contained colour pigments. It was, therefore, concluded that pigment transfer through mosquito handling was unlikely to bias the results and no correction was needed.

\section{Natural breeding sites vs. pupae collection}

The basic units over natural breeding sites marked an average of $0.6 \mathrm{An}$. gambiae s.l. per trap per day, with 15/15 mosquitoes being marked. The marking units that contained collected pupae marked an average of 4.4 An. gambiae s.l. per day, with 110/110 being marked. The latter method was, therefore, taken forward to the MMRR trial.

\section{MMRR field trial \\ Trapping}

A total of 5116 mosquitoes were caught and identified during the 12 days of trapping. Table 1 summarizes the data by trap type, mosquito family and sex. Of the 770 Anopheles mosquitoes captured, 8 were morphologically identified as An. funestus s.l. and the remaining were $A n$. gambiae s.l. Figure 5 shows Anopheline numbers by trap.

\section{Marking and recapture}

502 mosquitoes emerged from the marking unit over five marking days (Table 2). A correction factor of 0.86 , based on the average marking success in the semi-field experiment, was used to predict the number of marked mosquitoes. Of the 432 An. gambiae s.l. predicted to be marked, 41 An. gambiae s.l. were recaptured giving an overall recapture rate of $9.5 \%$. If a $50: 50$ sex ratio is 
Table 1 Summary of the trapping results during the MMRR study

\begin{tabular}{|c|c|c|c|c|}
\hline & \multicolumn{4}{|c|}{ Anopheline } \\
\hline & \multicolumn{2}{|c|}{ CDC-LT } & \multicolumn{2}{|l|}{$\mathrm{RBu}$} \\
\hline & Male & Female & Male & Female \\
\hline Total & 7 & 632 & 60 & 71 \\
\hline Total/trap & 0.32 & 31.60 & 2.00 & 2.37 \\
\hline Total/trap/night & 0.02 & 1.66 & 0.11 & 0.12 \\
\hline
\end{tabular}

assumed, then the recapture rate for females was $16.7 \%$ but only $1.9 \%$ for males. There were variations between the colour cohorts ranging from a $4.1 \%$ recapture success with the orange cohort to $30.9 \%$ recapture success with the green cohort.

\section{Daily survival probability}

Data from each colour cohort was combined to estimate the daily survival probability of the local female $A n$. gambiae s.l. population. As there where eight recapture days following the final marking day survival rates were calculated for days 1-8 after marking. Insufficient data were available to measure male survival. The exponential model was fitted to the $\log _{10}(x+1)$ number of marked mosquitoes recaptured against the days after marking (Fig. 7). The daily survival probability of female An. gambiae s.l. was 0.87 (95\% CI $0.69-1.10)$. This equates to a life expectancy of 7.2 days.

\section{Mean distance travelled}

The distribution of recaptured mosquitoes by distance is shown in Fig. 8. The MDT, which accounts for sampling effort by distance was $579 \mathrm{~m}$ (95\% CI 521-636 m) for female An. gambiae s.l. of any age. Using only female
An. gambiae s.l. recaptured up to 3 days after marking, the "first flight" MDT travelled was $597 \mathrm{~m}$ (95\% CI 509-685 m). Insufficient data were available to measure male MDT, however, the maximum male flight distance observed was $645 \mathrm{~m}$.

\section{Discussion}

This study demonstrates the successful use of a selfmarking device in an MMRR study with African malaria vectors. The self-marking method described here has the demonstrated potential use as means to measure the dispersal and survival of wild African malaria vectors. Studies of this kind will provide much needed parameter estimates for malaria transmission models [42] and allow the assessment of novel control tools. While many other

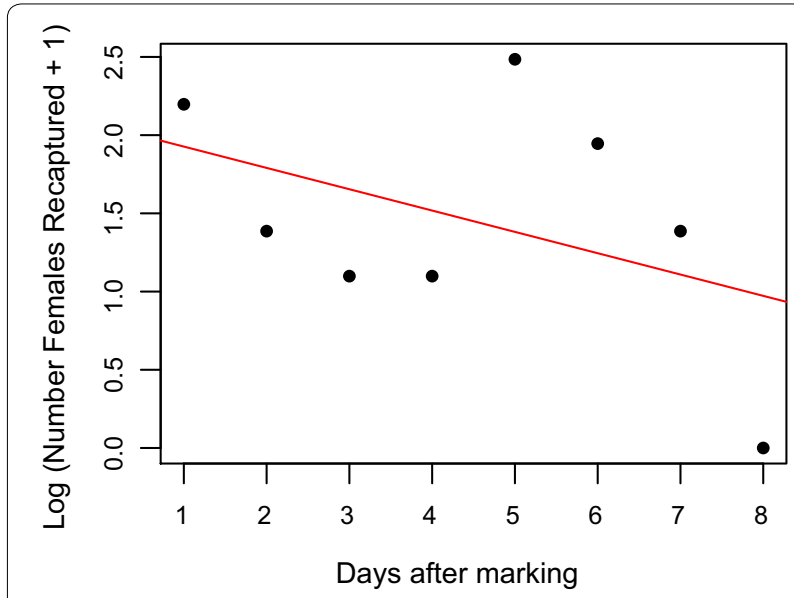

Fig. 7 Daily survival of female An. gambiae s.l. in Yombo, Tanzania. Log transformed number of recaptured female An. gambiae s.l. by days after marking. The fitted exponential model predicting a daily survival probability of 0.87

Table 2 Summary of the marking and recapture data

\begin{tabular}{|c|c|c|c|c|c|c|c|c|c|c|c|}
\hline \multirow[t]{2}{*}{ Dates } & \multicolumn{5}{|c|}{$\begin{array}{l}\text { Pupae emerged and approximate number of mosquitoes } \\
\text { marked }\end{array}$} & \multicolumn{6}{|c|}{ Number and proportion of marked recaptured mosquitoes } \\
\hline & Colour & $\begin{array}{l}\text { Number } \\
\text { emerged }\end{array}$ & $\begin{array}{l}\text { Number } \\
\text { marked }^{\text {a }}\end{array}$ & Male $^{\text {b }}$ & Female & Total & $\%$ & Female & $\%$ & Male & $\%$ \\
\hline 29/03/17 & Pink & 30 & 26 & 13 & 13 & 4 & 15.38 & 4 & 30.77 & 0 & 0 \\
\hline $30 / 03 / 17$ & Blue & 165 & 142 & 71 & 71 & 9 & 6.34 & 7 & 9.86 & 2 & 2.82 \\
\hline $31 / 03 / 17$ & Orange & 200 & 172 & 86 & 86 & 7 & 4.07 & 4 & 4.65 & 2 & 2.33 \\
\hline $01 / 04 / 17$ & Green & 64 & 55 & 27.5 & 27.5 & 17 & 30.91 & 17 & 61.82 & 0 & 0 \\
\hline \multirow[t]{2}{*}{$02 / 04 / 17$} & Yellow & 43 & 37 & 18.5 & 18.5 & 4 & 10.81 & 4 & 21.62 & 0 & 0 \\
\hline & Totals & 502 & 432 & 216 & 216 & 41 & 9.49 & 36 & 16.67 & 4 & 1.85 \\
\hline
\end{tabular}

The number of mosquitoes emerged through the unit was calculated from the number of pupae placed underneath the marking device and removing the number that remained the following day

a To calculate the number marked, a correction factor of 0.86 was applied to account for the marking success of the unit as observed in previous semi-field studies

b A 50:50 sex ratio of pupae is assumed to estimate the number of mosquitoes of each sex marked. An overall marking rate is given as well as data for each colour pigment for both sexes 


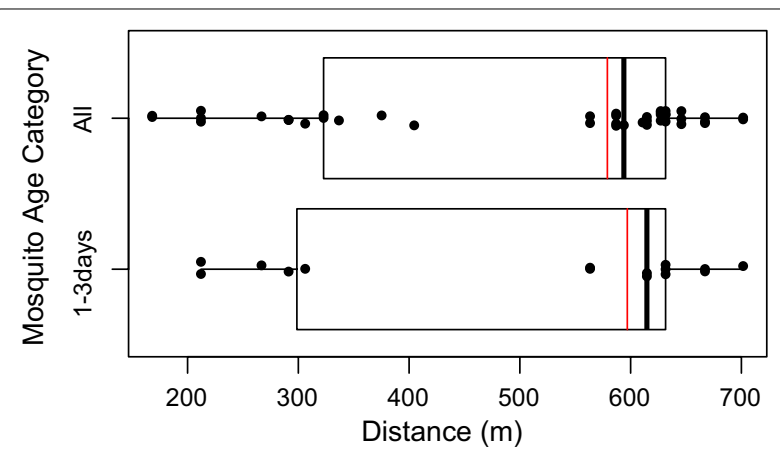

Fig. 8 Boxplots visualizing the distribution of recaptured mosquitoes by distance. The red line indicates the MDT which includes a correction for sampling effort over distance. All mosquitoes captured $(n=41)$ are represented in the top boxplot with only mosquitoes ages 3-days or less $(n=15)$ represented in the bottom boxplot. Dots indicate individual mosquitoes

methods for MMRR studies are available, the method evaluated minimizes the effect of human interference on the survival and dispersal of wild Anopheles mosquitoes.

\section{Pigment load and survival}

In the current study, a negative effect of the pigment on the survival of laboratory-reared anopheles mosquitoes was not observed which is in agreement with Niebylski and Meek's observation when using a self-marking device for Culex mosquitoes [27]. They attributed this to the fact the self-marking unit results in a relatively low pigment load on the mosquito (5-15 pigment particles) and mainly on the legs, abdomen and thorax area-similar pigment loads on were observed on the laboratoryreared and wild Anopheles recaptured in this study. This is in contrast to traditional dusting methods that apply a large pigment load, often covering wings and the sensory organs on the head, potentially impacting survival [7]. Fluorescent pigments could also impact a mosquito's host-seeking response and while the current evidence suggest the behaviour of An. gambiae s.s. is not affected by marking powders [24], further studies in this area are needed. The drawback to the lighter pigment load is that it is less obvious to the naked eye and it is more difficult to distinguish between the different pigment colours. UV torches and microscopes are, therefore, essential in identifying marked mosquitoes, which increases costs, time and workload for identification.

\section{Benefits of the self-marking unit}

A self-marking unit has clear benefits of reducing the man-power involved in marking and eliminates any human-handling that may be detrimental to mosquito survival or natural behaviour. Ethically, it is a preferable method because there are no additional mosquitoes being added to the population and no additional genetic material. By marking field caught mosquitoes as they emerge from pupae with the current device it is possible to know the exact age of a marked mosquito when it is recovered, providing information that is rarely known in MMRR studies with wild mosquitoes.

Release methods may have important implications for MMRR studies but are often overlooked. It has been suggested that some mosquitoes memorize their home range and establish flight paths $[17,43]$ and, therefore, using laboratory-reared mosquitoes or releasing adult caught mosquitoes in areas away from their origin, may misrepresent true dispersal. Allowing mosquitoes to emerge close to their breeding site and disperse in their own time removes this stress factor and any arbitrary effects of release point and time on their behaviour.

\section{Study limitations}

In the semi-field experiments around $14 \%$ of mosquitoes emerging from the device did not pick up the fluorescent pigment. It was possible to correct for this during analysis, however, complete marking success would be preferred. Niebylski and Meek [27] observed 100\% marking success with their device and this may be due to the cloth used: they impregnated cheesecloth with pigment whereas in the current study, a more closely woven white cotton fabric was used as it was available locally. Modifications to the device could be made using materials to increase pigment transfer, for example, electrostatic gauze has been previously shown to mark mosquitoes with pigment after brief contact [44].

During the semi-field experiments, pigment transfer between mosquitoes was observed when manually aspirating them in groups. Due to a small diameter of the aspirator, mosquitoes occasionally get bunched together which may have caused the transfer of pigment. CDCLT or Prokopack aspirators have much larger trapping containers and so mosquitoes are less likely to come into direct contact with each other and for pigment transfer to occur. Previous studies have observed low levels of pigment transfer when dusted mosquitoes are held together [7], but transfer has not been observed between mating pairs $[7,27]$. Here it was observed that recapture methods can also cause pigment transfer and so should be considered when selecting recapture methods in a MMRR trial. During the trapping phase of the MMRR study mosquitoes carrying the same colour pigment was observed in the same trap on the same night on four occasions. This indicates the mosquitoes emerging on the same night arrived at the same house independently, however, contamination cannot be ruled out. In the semi-field experiments a small number of control mosquitoes were 
identified to be carrying colour pigment which could be due to contamination through forceps or microscope-it is, therefore, important to keep equipment scrupulously clean during mark-recapture experiments. It was also observed that swapping the colour grids each day was quite messy and a small amount of pigment from the previous day remained on the marking frame and surrounding area. This could be overcome by making more marking frames to keep the colours independent. On the rare occasion were two colour pigments on the same mosquito were observed, it was assumed that the mosquito emerged on the day of the most recent colour.

It was previously noted that a limitation of the selfmarking device over a natural breeding site was that the number of mosquitoes emerging and the time of emergence could not be determined [45]. A possible solution would be to use an infrared counting system like the Biogents BG-Counter trap (Biogents AG, Regensburg, Germany) to count mosquitoes exiting the marking unit. However, to gain accurate estimates of the numbers emerging from the device, it is also possible to place pupae collected from several closely located breeding sites underneath the marking device and count pupal emergence as was done in this study and in a separate study with Aedes albopictus, [41]. Preliminary evaluations of the units indicated that it was impractical to mark the vectors of interest emerging from their natural breeding site. The basic marking units covered a breeding site of $0.25 \mathrm{~m}^{2}$ and marked 0.6 mosquitoes per day and so, assuming an equal emergence rate across the breeding site, an area roughly $20.8 \mathrm{~m}^{2}$ would need to be covered by emergence markers ( 84 self-marking units) to mark a minimum of 50 mosquitoes a day. This was not feasible in the breeding site under investigation and is unlikely for other malaria vector breeding sites were emergence rates might be even lower. The weekly emergence rate of anopheline mosquitoes in The Gambia has been estimated as 0.56 mosquitoes per $\mathrm{m}^{2}$ per week [46] and a study in the western Kenyan highlands estimated the emergence of An. gambiae as 1.82 per $\mathrm{m}^{2}$ per week [47]. In the current study, collecting pupae and stage four larvae from the surrounding area was very successful; however, the methodology is still dependent on there being relatively productive breeding sites available. Emergence rates from breeding sites will vary due to host of environmental factors and will vary dramatically between mosquito species. Before considering the use of the marking unit it is recommended that an entomological survey be conducted to determine if mosquitoes will emerge in sufficient numbers to be marked.

While this is certainly a limitation in areas with low mosquito numbers, overall the self-marking system provides a useful non-invasive alternative to traditional
MMRR for measuring wild mosquito bionomics. The marking unit has also now been used in a MMRR study in Switzerland with Aedes albopictus [48].

\section{Mosquito survival estimates}

Due to the small sampling area, clustering of houses in the MMRR study and short collection period, the distance and survival estimates calculated have to be interpreted with caution. MDT estimates are highly correlated to sampling area in MMRR studies [8] and survival estimates are influenced by mosquitoes leaving the study area. Despite this, the estimate of 0.87 as the daily survival of probability of female An. gambiae s.l. was similar to that found in previous studies. Gillies estimated the daily survival of An. gambiae to be 0.841 in an area of Tanzania slightly further inland. Other studies predicted daily survival to be $0.80-0.88$ in Burkina Faso [18], 0.80 in Mali [11] and estimates ranging from 0.78 in another Tanzanian study [10] up to 0.95 in coastal Kenya [12]. The few estimates for An. funestus are more widely dispersed and range from a daily survival rate of 0.63 [10] to 0.837 [43] and up to 0.96 [12].

\section{Mosquito dispersal studies}

Of the studies that previously measure dispersal in $A n$. gambiae s.l., Costantini et al. sampled an area of similar size to the current study and estimated the daily dispersal of female An. gambiae s.l. to be 350-650 m which is line with $579 \mathrm{~m}$ overall MDT and $597 \mathrm{~m}$ "first flight" MDT measured here. Gillies on the other hand sampled up to $3.62 \mathrm{~km}$ away from the release point and estimated the mean dispersal distance (unadjusted) of female mosquitoes to be 1.02 and $1.58 \mathrm{~km}$ depending on their release point, in the centre or periphery of a village respectively. These distances were calculated over 23 days and, therefore, could include back and forth flight. To account for this, Gillies also looked at the dispersal after 1 day and found it to be $720 \mathrm{~m}$ in the central area which is similar to both the estimates of Costantini et al. [18] and those observed in the current study. While the calculation of MDT corrects for sampling effort over distance it assumes all locations are equally attractive to mosquitoes. Anthropophilic mosquitoes are attracted to areas of high population density $[9,49]$, and mosquito traps are necessarily differentially located in or around houses. The implications of this for estimation of MDT would merit further theoretical investigation. Gillies also suggests mosquito dispersal is highly influenced by the local topographical factors and therefore cautions against making generalizations outside a particular study setting. To gain a clearer understanding of mosquito dispersal there is a need for future MMRR studies to determine the 
factors influencing mosquito dispersal rather than just describing dispersal distances. As the current study was a proof of concept it was restricted in size; however, the self-marking unit has since been used in a large scale MMRR study (Saddler et al. in preparation) that will further add to the mosquito biology knowledge base.

\section{Conclusions}

Despite the importance of mosquitoes for the transmission of malaria, there are relatively few empirical studies investigating key entomological parameters in wild mosquitoes. MMRR studies still have an important role in obtaining field estimates; and although there are a variety of methods available to mark mosquitoes, the self-marking unit described here has several logistical, ethical and biological benefits. The unit successfully marked wild An. gambiae s.l. males and females in sufficiently large numbers to justify its use in MMRR studies. The estimated daily survival probability of $A n$. gambiae s.l. was 0.87 and mean dispersal distance was $597 \mathrm{~m}$. These benefits may encourage further MMRR studies, allowing more accurate modelling and localized predictions of malaria transmission. In addition, the technique is simple enough to be used in vector control studies where population age and dispersal are important, including testing new vector control tools such as spatial repellents, gene drive mosquitoes and ATSB.

\section{Supplementary information}

Supplementary information accompanies this paper at https://doi. org/10.1186/s12936-019-3077-3.

Additional file 1. MMRR field trial dataset. Contains data on individual recaptured mosquitoes, pupae numbers and mosquito trapping totals (marked and unmarked).

\section{Abbreviations}

ALE: average life expectancy; ATSBs: attractive targeted sugar baits; CDC-LT: Centers for Disease Control and Prevention light trap; CF: correction factor; EAT: East African Time; ER: estimated recapture; GLMM: Generalized Linear Mixed Effects Models; IHI: Ifakara Health Institute; MDT: mean distance travelled; MMRR: mosquito mark-release-recapture; RBu: resting buckets.

\section{Acknowledgements}

We thank all the technicians at Ifakara Health Institute and the volunteers from Yombo village who participated in the mosquito collections. Dr K Kreppel acknowledges support from the DELTAS Africa Initiative (Afrique OneASPIRE/DEL-15-008). Sarah J Moore was funded through a grant from the Innovative Vector Control Consortium (IVCC). IVCC would like to acknowledge that source funding for the 'Push-Pull' project came from the Bill \& Melinda Gates Foundation and UKaid. Nakul Chitnis and Thomas Smith were funded by the Bill and Melinda Gates Foundation with Grant OPP1032350. The authors are grateful to Pie Müller and Laura Vavassori for helpful discussions on marking technology and analysis.

\section{Authors' contributions}

SJM, TAS, NC conceived the study; AS, JDM designed the modified marking unit; AS, MMT, KSK performed the data collection; AS wrote the manuscript; KSK, SJM, AD, TAS and NC critically revised the manuscript. All authors read and approved the final manuscript.

\section{Funding}

This work was supported by the Swiss National Science Foundation, Grant Number 320030_163473, "Movement rates of African malaria vectors and their implications in models of vector control interventions".

\section{Availability of data and materials}

Data generated and analysed during this study are included in this published article and its Additional file 1.

\section{Ethics approval and consent to participate}

Full ethical approval was obtained from ethical review committees at Ifakara Health Institute (IHI/IRB/No: 016-2016) and the National Institute for Medical Research (NIMR/HQ/R.8c/Nol. IX/2392). Households used for mosquito collections were recruited upon written informed consent of the household head.

\section{Consent for publication}

All authors read and approved the final version of the manuscript.

\section{Competing interests}

The authors declare that they have no competing interests.

\section{Author details}

${ }^{1}$ Ifakara Health Institute, Environmental Health and Ecological Sciences, P.O. Box 74, Bagamoyo, Tanzania. ${ }^{2}$ Swiss Tropical \& Public Health Institute, Socinstrasse 57, 4051 Basel, Switzerland. ${ }^{3}$ University of Basel, Petersplatz 1, 4001 Basel, Switzerland. ${ }^{4}$ Nelson Mandela African Institution of Science and Technology, P.O. Box 447, Tengeru, Tanzania.

Received: 1 October 2019 Accepted: 14 December 2019

Published online: 23 December 2019

\section{References}

1. Achee NL, Bangs MJ, Farlow R, Killeen GF, Lindsay S, Logan JG, et al. Spatial repellents: from discovery and development to evidence-based validation. Malar J. 2012;11:164.

2. Maia MF, Kliner M, Richardson M, Lengeler C, Moore SJ. Mosquito repellents for malaria prevention. Cochrane Database Syst Rev. 2018;2:CD011595.

3. Marshall JM, White MT, Ghani AC, Schlein Y, Muller GC, Beier JC. Quantifying the mosquito's sweet tooth: modelling the effectiveness of attractive toxic sugar baits (ATSB) for malaria vector control. Malar J. 2013;12:291.

4. Qualls WA, Muller GC, Traore SF, Traore MM, Arheart KL, Doumbia S, et al. Indoor use of attractive toxic sugar bait (ATSB) to effectively control malaria vectors in Mali, West Africa. Malar J. 2015;14:301.

5. James S, Collins FH, Welkhoff PA, Emerson C, Godfray HCJ, Gottlieb M, et al. Pathway to deployment of gene drive mosquitoes as a potential biocontrol tool for elimination of malaria in sub-Saharan Africa: recommendations of a Scientific Working Group. Am J Trop Med Hyg. 2018;98(Suppl 6):1-49.

6. Epopa PS, Millogo AA, Collins CM, North A, Tripet F, Benedict MQ, et al. The use of sequential mark-release-recapture experiments to estimate population size, survival and dispersal of male mosquitoes of the Anopheles gambiae complex in Bana, a west African humid savannah village. Parasit Vectors. 2017;10:376.

7. Service M. Mosquito ecology: field sampling methods. 2nd ed. London: Chapman \& Hall; 1993.

8. Guerra CA, Reiner RC Jr, Perkins TA, Lindsay SW, Midega JT, Brady OJ, et al. A global assembly of adult female mosquito mark-release-recapture data to inform the control of mosquito-borne pathogens. Parasit Vectors. 2014;7:276.

9. Gillies MT. Studies on the dispersion and survival of Anopheles gambiae Giles in East Africa, by means of marking and release experiments. Bull Entomol Res. 1961;52:99. 
10. Takken W, Charlwood JD, Billingsley PF, Gort G. Dispersal and survival of Anopheles funestus and A. gambiae s.l. (Diptera: Culicidae) during the rainy season in southeast Tanzania. Bull Entomol Res. 1998;88:561.

11. Touré YT, Dolo G, Petrarca V, Traoré SF, Bouaré M, Dao A, et al. Markrelease-recapture experiments with Anopheles gambiae s.I. in Banambani village, Mali, to determine population size and structure. Med Vet Entomol. 1998;12:74-83.

12. Midega JT, Mbogo CM, Mwnambi H, Wilson MD, Ojwang G, Mwangangi JM, et al. Estimating dispersal and survival of Anopheles gambiae and Anopheles funestus along the Kenyan coast by using mark-releaserecapture methods. J Med Entomol. 2007;44:923-9.

13. Lines JD, Lyimo EO, Curtis CF. Mixing of indoor- and outdoor-resting adults of Anopheles gambiae Giles s.I. and A. funestus Giles (Diptera: Culicidae) in coastal Tanzania. Bull Entomol Res. 1986;76:171.

14. Baber I, Keita M, Sogoba N, Konate M, Diallo MB, Doumbia S, et al. Population size and migration of Anopheles gambiae in the Bancoumana region of Mali and their significance for efficient vector control. PLOS ONE. 2010;5:e10270.

15. Mnzava AE, Rwegoshora RT, Wilkes TJ, Tanner M, Curtis CF. Anopheles arabiensis and An. gambiae chromosomal inversion polymorphism, feeding and resting behaviour in relation to insecticide house-spraying in Tanzania. Med Vet Entomol. 1995;9:316-24.

16. Quiñones ML, Lines JD, Thomson MC, Jawara M, Morris J, Greenwood BM. Anopheles gambiae gonotrophic cycle duration, biting and exiting behaviour unaffected by permethrin-impregnated bednets in The Gambia. Med Vet Entomol. 1997;11:71-8.

17. McCall PJ, Mosha FW, Njunwa KJ, Sherlock K. Evidence for memorized site-fidelity in Anopheles arabiensis. Trans R Soc Trop Med Hyg. 2001;95:587-90.

18. Costantini C, Li SG, Della Torre A, Sagnon N, Coluzzi M, Taylor CE. Density, survival and dispersal of Anopheles gambiae complex mosquitoes in a west African Sudan savanna village. Med Vet Entomol. 1996;10:203-19.

19. Kang SY, Battle KE, Gibson HS, Cooper LV, Maxwell K, Kamya M, et al. Heterogeneous exposure and hotspots for malaria vectors at three study sites in Uganda. Gates Open Res. 2018;2:32.

20. WHO. Design of epidemiological trials for vector control products, Report of a WHO Expert Advisory Group, Chateau de Penthes, Geneva, 24-25 April 2017. https://www.who.int/neglected_diseases/vecto r_ecology/resources/WHO_HTM_NTD_VEM_2017.04/en/. Accessed 10 July 2019.

21. Silver JB. Mosquito ecology: field sampling methods. 3rd ed. Amsterdam: Springer; 2008.

22. Benedict M, Charlwood J, Harrington L, Philip Lounibos L, Reisen W Tabachnick W. Guidance for evaluating the safety of experimental releases of mosquitoes, emphasizing mark-release-recapture techniques. Vector Borne Zoonotic Dis. 2018;18:39-48.

23. Curtis CF, Rawlings P. A preliminary study of dispersal and survival of Anopheles culicifacies in relation to the possibility of inhibiting the spread of insecticide resistance. Ecol Entomol. 1980;5:11-7.

24. Verhulst NO, Loonen JA, Takken W. Advances in methods for colour marking of mosquitoes. Parasit Vectors. 2013;6:200.

25. Dickens BL, Brant HL. Effects of marking methods and fluorescent dusts on Aedes aegypti survival. Parasit Vectors. 2014;7:65.

26. Rawlings $P$, Curtis CF, Wickramasinghe MB, Lines J. The influence of age and season on dispersal and recapture of Anopheles culicifacies in Sri Lanka. Ecol Entomol. 1981;6:307-19.

27. Niebylski ML, Meek CL. A self-marking device for emergent adult mosquitoes. J Am Mosquito Contr. 1989;5:86-90.

28. Lorenz LM, Overgaard HJ, Massue DJ, Mageni ZD, Bradley J, Moore $J \mathrm{D}$, et al. Investigating mosquito net durability for malaria control in Tanzania-attrition, bioefficacy, chemistry, degradation and insecticide resistance (ABCDR): study protocol. BMC Public Health. 2014;14:1266.

29. Temu EA, Minjas JN, Coetzee M, Hunt RH, Shiff CJ. The role of four anopheline species (Diptera: Culicidae) in malaria transmission in coastal Tanzania. Trans R Soc Trop Med Hyg. 1998;92:152-8.
30. Andres M, Lorenz LM, Mbeleya E, Moore SJ. Modified mosquito landing boxes dispensing transfluthrin provide effective protection against Anopheles arabiensis mosquitoes under simulated outdoor conditions in a semi-field system. Malar J. 2015;14:255.

31. Okumu FO, Moore J, Mbeyela E, Sherlock M, Sangusangu R, Ligamba $G$, et al. A modified experimental hut design for studying responses of disease-transmitting mosquitoes to indoor interventions: the Ifakara experimental huts. PLoS ONE. 2012;7:e30967.

32. Vazquez-Prokopec GM, Galvin WA, Kelly R, Kitron U. A new, cost-effective, battery-powered aspirator for adult mosquito collections. J Med Entomol. 2009;46:1256-9.

33. QGIS Delopment Team. QGIS Geographic Information System. Open Source Geospatial Foundation. 2019.

34. Kreppel KS, Johnson PCD, Govella NJ, Pombi M, Maliti D, Ferguson HM. Comparative evaluation of the Sticky-Resting-Box-Trap, the standardised resting-bucket-trap and indoor aspiration for sampling malaria vectors. Parasit Vectors. 2015;8:462.

35. R Core Team. R: a language and environment for statistical computing. Vienna, Austria. 2018.

36. Therneau T. coxme: mixed effects Cox MODELS. 2019. https://repo. bppt.go.id/cran/web/packages/coxme/vignettes/coxme.pdf.

37. Bates D, Mächler M, Bolker B, Walker S. Fitting linear mixed-effects models using Ime4. J Stat Softw. 2015;67:1-48.

38. Hothorn T, Bretz F, Westfall P. Simultaneous inference in general parametric models. Biom J. 2008;50:346-63.

39. Lillie TH, Marquardt WC, Jones RH. The flight range of Culicoides variipennis (Diptera: Ceratopogonidae). Can Entomol. 1981;113:419-26.

40. Morris CD, Larson VL, Lounibos LP. Measuring mosquito dispersal for control programs. J Am Mosquito Contr. 1991;7:608-15.

41. Niebylski ML, Craig GB. Dispersal and survival of Aedes albopictus at a scrap tire yard in Missouri. J Am Mosquito Contr. 1994;10:339-43.

42. Endo N, Eltahir EAB. Modelling and observing the role of wind in Anopheles population dynamics around a reservoir. Malar J. 2018;17:48.

43. Charlwood JD, Vij R, Billingsley PF. Dry season refugia of malaria-transmitting mosquitoes in a dry savannah zone of east Africa. Am J Trop Med Hyg. 2000;62:726-32.

44. Andriessen R, Snetselaar J, Suer RA, Osinga AJ, Deschietere J, Lyimo IN, et al. Electrostatic coating enhances bioavailability of insecticides and breaks pyrethroid resistance in mosquitoes. Proc Natl Acad Sci USA. 2015;112:12081-6.

45. Ciota AT, Drummond CL, Ruby MA, Drobnack J, Ebel GD, Kramer LD. Dispersal of Culex mosquitoes (Diptera: Culicidae) from a wastewater treatment facility. J Med Entomol. 2012;49:35-42.

46. Fillinger U, Sombroek H, Majambere S, van Loon E, Takken W, Lindsay SW. Identifying the most productive breeding sites for malaria mosquitoes in The Gambia. Malar J. 2009;8:62.

47. Kweka EJ, Zhou G, Lee M-C, Gilbreath TM, Mosha F, Munga S, et al. Evaluation of two methods of estimating larval habitat productivity in western Kenya highlands. Parasite Vectors. 2011:4:110.

48. Vavassori L, Saddler A, Müller P. Active dispersal of Aedes albopictus: a mark-release-recapture study using self-marking units. Parasit Vectors. 2019;12:583.

49. Smith T, Charlwood JD, Takken W, Tanner M, Spiegelhalter DJ. Mapping the densities of malaria vectors within a single village. Acta Trop. 1995;59:1-18.

50. OpenStreetMap Contributors: OpenStreetMap. 2019. https://www.opens treetmap.org/. Accessed 10 July 2019.

\section{Publisher's Note}

Springer Nature remains neutral with regard to jurisdictional claims in published maps and institutional affiliations. 